\title{
Robust Deadbeat Continuous-Time Observer Design Based on Modulation Integrals
}

\author{
Gilberto Pin ${ }^{\mathrm{a}}$, Boli Chen ${ }^{\mathrm{b}}$, Thomas Parisini ${ }^{\mathrm{b}, \mathrm{c}, \mathrm{d}}$ \\ ${ }^{\text {a }}$ Electrolux Italia S.p.A, Italy \\ ${ }^{\mathrm{b}}$ Dept. of Electrical and Electronic Engineering, Imperial College London, UK \\ ${ }^{\mathrm{c}}$ Dept. of Engineering and Architecture, University of Trieste, Italy \\ ${ }^{\mathrm{d}}$ KIOS Research and Innovation Centre of Excellence, University of Cyprus
}

\begin{abstract}
In this paper, the state estimation problem of linear continuous-time systems is dealt with by a non-asymptotic state observer, which allows the state estimation error to decay within an arbitrarily-small finite time without resorting to high-gain injection. By processing the measured input and output signals through modulation integrals, a number of auxiliary signals not affected by the initial conditions are obtained, from which the system state can be computed by simple algebra. The problem of internal instability of modulation integrals is addressed by resorting to a periodic rescaling mechanism that prevents error accumulation and singularities. We show that the combination of modulation integrals with periodic rescaling can be implemented as a jump-linear system. The robustness of the devised method with respect to additive measurement perturbations on the system's input/output is characterized by Input-to-State Stability arguments.
\end{abstract}

\section{Introduction}

The problem of dynamic state estimation is very important, both for monitoring hidden variables not accessible by instrumentation and for output feedback control systems design. Standard state observers for linear time invariant systems are usually characterized by asymptotic convergence properties (see $[1,2]$ ). However, in some applications, such as fault isolation or changepoint detection, the state estimates are often required to converge in a neighborhood of the true values within a pre-determined finite (and possibly very small) time. This behavior is referred to as deadbeat convergence. Several approaches for deadbeat state estimation are already available in the literature. Among them, the Sliding Mode (SM) observer appears as one of the most used techniques due to its ease of implementation. Notably, the SM conventional observer suffers from a discontinuous high-gain output injection (chattering), which may prevent its applicability in a noisy environment. More-

\footnotetext{
^ This work has been supported by the European Union's Horizon 2020 Research and Innovation Program under the grant agreement No. 739551 (KIOS CoE).

Email addresses: gilbertopin@alice.it (Gilberto Pin), boli.chen10@imperial.ac.uk (Boli Chen), t.parisini@gmail.com (Thomas Parisini ).
}

over, these observers can only guarantee the semi-global stability of the estimation-error dynamics. The chattering phenomenon can be mitigated by the use of higherorder SM approaches (see [3-6]) that, in addition, can be proven to achieve global convergence at the cost of increased complexity. Very recently, the homogeneity concept has been used to design high-gain observers that are able to converge in finite-time $[7,8]$.

Several methods alternative to the SM observer have been conceived to address the problem of deadbeat state estimation in order to the remove the need for highgain output injection. For instance, the delay-based filters have been originally proposed in [10] and further developed in [11]. Clearly the use of delay operators calls for large memory storage at the implementation stage and this point represents a major obstacle for the practical application. Another alternative is the impulsive observer (IO) that is based on impulsive innovation updates [12]. While the original formulation of the method is non-minimal, the IO has been subsequently modified to reduce the dimensionality of the observer at the price of non-instantaneous convergence [13]; indeed, the deadbeat performance is achieved in a finite number of impulsive updates. Another class of deadbeat state estimators is represented by the moving-horizon and convolutional observers, that are described respectively in [14] 
and [15] and are characterized by need to solve repeated dynamic optimization problems on-line. In a recent paper [16], a finite-time observer is designed for linear system via the implicit Lyapunov function approach $[17,9]$. This observer has an advantageous feature of being able to converge after a fixed-time interval defined a priori.

In the present brief, a finite-time observer for continuoustime linear systems is proposed by resorting to modulation integrals. The concept originally stems from the kernel based observer initially proposed by the authors in [18]. However, the complexity is significantly reduced by replacing the bivariate kernels of [18] with the newly designed univariate modulating functions (MFs) whereas comparable performance is achieved. Compared to the work presented in [21] that is also based on univariate MFs, the proposed MFs are characterized by onesided boundary conditions. The internal variables of the modulation integral observer are periodically rescaled to avoid error accumulation in noisy environments. In contrast to the approach proposed in [20] that requires periodic resetting to avoid numerical issues due to internal instability, the proposed rescaling strategy does not completely discard old data, but acts as a sort of forgetting factor which allows to maintain most of the old information.

The paper is organized as follows: the problem is formulated in Section 2 and in Section 3, the modulation integral observer is introduced. Then, Section 4 illustrates a rescaling mechanism introduced to avoid divergence phenomena in the integral operators and Section 5 provides a thorough theoretical robustness analysis in which the rescaling technique is exploited. Furthermore, Section 6 provides insight on the choice of MFs. Extensive simulation results are provided in Section 7 and Section 8 draws some concluding remarks. Throughout the paper, $\mathbb{R}, \mathbb{R}_{\geq 0}$ and $\mathbb{R}_{>0}$ will denote the real, the non-negative real and the strict positive real sets of numbers, respectively. Similarly, $\mathbb{Z}, \mathbb{Z}_{>0}$ and $\mathbb{Z}_{>0}$ will denote respectively the integer, the non-negative integer and the strict positive integer sets of numbers. Moreover, we denote by $w^{(i)}$ the $i$-th order derivative signal of $w(t) \in \mathbb{R}, \forall t \geq 0$, which is assumed to be $i$-th order differentiable.

\section{Problem Statement and Preliminaries}

Consider the following single-input single-output (SISO) continuous-time linear system

$$
\left\{\begin{array}{l}
\dot{\mathbf{x}}(t)=\mathbf{A} \mathbf{x}(t)+\mathbf{b} u(t) \\
y(t)=\mathbf{c}^{\top} \mathbf{x}(t)
\end{array}\right.
$$

where $\mathbf{x} \in \mathbb{R}^{n}, u \in \mathbb{R}, y \in \mathbb{R}$. The pair $u(t), y(t)$ is assumed to be measurable at any time $t \geq 0$, while their time-derivatives are not assumed to be available. The matrices $\mathbf{A}, \mathbf{b}, \mathbf{c}^{\top}$ are known constant matrices, and the pair $\left(\mathbf{A}, \mathbf{c}^{\top}\right)$ is fully observable. Our objective consists in providing a non-asymptotic (fast) estimate of the state $\mathbf{x}(t)$ of system (1), by suitably processing the input and output signals $u(t)$ and $y(t)$, in such a way that the unknown value of the initial conditions $\mathbf{x}_{0}$ does not affect the transient behavior of the estimator.

Owing to the observability of (1), there exists a linear change of coordinates $\mathbf{z}(t)=\mathbf{T} \mathbf{x}(t)$ with $\mathbf{z} \in \mathbb{R}^{n}$, such that the dynamics of $\mathbf{z}(t)$ is governed by the following state-space realization:

$$
\left\{\begin{array}{l}
\dot{\mathbf{z}}(t)=\mathbf{A}_{z} \mathbf{z}(t)+\mathbf{b}_{z} u(t) \\
y(t)=\mathbf{c}_{z}^{\top} \mathbf{z}(t), \quad t \in \mathbb{R}_{\geq 0}
\end{array}\right.
$$

where $\mathbf{z}(t) \triangleq\left[\begin{array}{llllll}z_{0}(t) & z_{1}(t) & \cdots & z_{r}(t) & \cdots & z_{n-1}(t)\end{array}\right]^{\top} \in \mathbb{R}^{n}$ is the system' state vector, while $\mathbf{b}_{z}=\mathbf{T b}, \mathbf{c}_{z}^{\top}=\mathbf{c}^{\top} \mathbf{T}^{-1}$ and $\mathbf{A}_{z}=\mathbf{T A T}^{-1} \in \mathbb{R}^{n \times n}$ are given by

$$
\mathbf{A}_{z}=\left[\begin{array}{ccccc}
a_{n-1} & 1 & 0 & \cdots & 0 \\
\vdots & \vdots & \ddots & \ddots & 0 \\
a_{1} & 0 & \cdots & 0 & 1 \\
a_{0} & 0 & \cdots & 0 & 0
\end{array}\right], \mathbf{b}_{z}=\left[\begin{array}{c}
0 \\
\vdots \\
b_{m-1} \\
\vdots \\
b_{0}
\end{array}\right], \mathbf{c}_{z}=\left[\begin{array}{c}
1 \\
0 \\
\vdots \\
0
\end{array}\right]
$$

with $m \in \mathbb{Z}_{>0}, n \in \mathbb{Z}_{>0}, m \leq n$. Thanks to the transformation given in (2) and (3), the estimation of $\mathbf{x}(t)$ can be addressed by estimating $\mathbf{z}(t)$ in the canonical system. For the sake of further discussion, it is worth to introduce the I/O differential model of (2):

$$
\mathcal{S}_{u \rightarrow y}:\left\{\begin{array}{l}
y^{(n)}(t)=\sum_{i=0}^{n-1} a_{i} y^{(i)}(t)+\sum_{k=0}^{m-1} b_{k} u^{(k)}(t) \\
y^{(i)}(0)=y_{0}^{(i)}, i \in\{0, \ldots, n-1\} \\
u^{(k)}(0)=u_{0}^{(k)}, k \in\{0, \ldots, m-1\}
\end{array}\right.
$$

The state-variables of the observer canonical realization $z_{r}(t), r \in\{0, \cdots, n-1\}$ can be expressed as a linear combination of the input-output derivatives:

$$
z_{r}(t)=y^{(r)}(t)-\sum_{j=0}^{r-1} a_{n-r+j} y^{(j)}(t)-\sum_{j=0}^{r-1+m-n} b_{n-r+j} u^{(j)}(t)
$$

where we have used the convention $\sum_{j=0}^{k}\{\cdot\}=0, \quad \forall k<0$. 


\section{The Modulation Integral Observer}

The modulation integral over the time interval $\left[t_{0}, t\right], 0 \leq t_{0} \leq t$ is defined as follows:

$$
\left[V_{\phi} w\right]\left(t \mid t_{0}\right) \triangleq \int_{t_{0}}^{t} \phi\left(\tau-t_{0}\right) w(\tau) d \tau, \quad t \in \mathbb{R}_{\geq t_{0}}
$$

where $\phi(\cdot) \in \mathscr{C}^{i}, \forall t \in \mathbb{R}_{\geq 0}$ is the MF. A compact version is also defined for the case when $t_{0}=0$

$$
\left[V_{\phi} w\right](t) \triangleq \int_{0}^{t} \phi(\tau) w(\tau) d \tau, \quad t \in \mathbb{R}_{\geq 0} .
$$

Lemma 3.1 (Modulated signal's derivative) For a given $i \geq 0$, consider a signal $w(\cdot) \in \mathscr{L}^{2}\left(\mathbb{R}_{\geq 0}\right)$ that admits an $i$-th order derivative in $\mathbb{R}_{\geq 0}$ and a function $\phi(\cdot) \in \mathscr{C}^{i}, \forall t \in \mathbb{R}_{\geq 0}$. Then, for all $t \in \mathbb{R}_{\geq 0}$, it holds that:

$$
\begin{aligned}
& {\left[V_{\phi} w^{(i)}\right](t)=\sum_{j=0}^{i-1}(-1)^{i-j-1} w^{(j)}(t) \phi^{(i-j-1)}(t)} \\
& +\sum_{j=0}^{i-1}(-1)^{i-j} w^{(j)}(0) \phi^{(i-j-1)}(0)+(-1)^{i}\left[V_{\phi^{(i)}} w\right](t)
\end{aligned}
$$

that is, $\left[V_{\phi} w^{(i)}\right](\cdot)$ can be expressed as a function of $w(\cdot)$ and its first $(i-1)$-th derivatives $w^{(1)}(\cdot), \ldots, w^{(i-1)}(\cdot)$.

Proof. Integrating by parts, we have:

$$
\begin{array}{r}
{\left[\mathcal{V}_{\phi} w^{(i)}\right](t)=\int_{0}^{t} \phi(\tau) w^{(i)}(\tau) d \tau=w^{(i-1)}(t) \phi(t)} \\
-u^{(i-1)}(0) \phi(0)-\int_{0}^{t} \phi^{(1)}(\tau) w^{(i-1)}(\tau) d \tau
\end{array}
$$

The integral operator on the right-hand side of (7) can be further split by parts:

$$
\begin{aligned}
-\int_{0}^{t} \phi^{(1)}(\tau) w^{(i-1)}(\tau) d \tau & =-w^{(i-2)}(t) \phi^{(1)}(t) \\
& +w^{(i-2)}(0) \phi^{(1)}(0)+\int_{0}^{t} \phi^{(2)}(\tau) w^{(i-2)}(\tau) d \tau
\end{aligned}
$$

Proceeding by induction we obtain

$$
\begin{gathered}
\int_{0}^{t} \phi(\tau) w^{(i)}(\tau) d \tau=\sum_{j=1}^{i}(-1)^{j+1} w^{(i-j)}(t) \phi^{(j-1)}(t)+ \\
\sum_{j=1}^{i}(-1)^{j} w^{(i-j)}(0) \phi^{(j-1)}(0)+(-1)^{i} \int_{0}^{t} \phi^{(i)}(\tau) w(\tau) d \tau
\end{gathered}
$$

that is, the function obtained by applying the modulation integral to the $i$-th derivative depends on lowerorder signal derivatives. The identity (6) can be verified by rearranging indexing of the summation in (8).

The following definition characterizes the MFs for which the transformed signal $\left[V_{\phi} w^{(i)}\right](t)$ in $(6)$ is independent from the initial values of the signal and its derivatives.

Definition 3.1 ( $i$-th order modulating function) Consider a function $\phi(\cdot) \in \mathscr{C}^{i}$; if for a given $i \geq 1, \phi(\cdot)$ and its derivatives verify the vanishing conditions

$$
\phi^{(j)}(0)=0, \forall j \in\{0, \ldots, i-1\},
$$

then $\phi(\cdot)$ it is called an $i$-th order MF. Some examples of admissible MFs will be given later in Section 6 .

Assuming that $\phi_{1}(\cdot)$ is an $n$-th order MF, by changing the integrand $w^{(i)}$ in (6) with $y^{(i)}$ and $u^{(k)}$ we respectively get:

$$
\begin{array}{r}
{\left[V_{\phi_{1}} y^{(i)}\right](t)=\sum_{j=0}^{i-1}(-1)^{i-1-j} y^{(j)}(t) \phi_{1}^{(i-j-1)}(t)} \\
+(-1)^{i}\left[V_{\phi_{1}^{(i)}} y\right](t), i \in\{1, \ldots, n-1\}
\end{array}
$$

and

$$
\begin{gathered}
{\left[V_{\phi_{1}} u^{(k)}\right](t)=\sum_{j=0}^{k-1}(-1)^{k-1-j} u^{(j)}(t) \phi_{1}^{(k-j-1)}(t)} \\
+(-1)^{k}\left[V_{\phi_{1}^{(k)}} u\right](t), k \in\{1, \ldots, m-1\}
\end{gathered}
$$

Considering the case $i=1$ in (10), changing the MF $\phi_{1}$ with $\phi_{1}^{(j)}, \forall j \in\{1, \ldots, n-1\}$, and then performing the substitution of $y^{(n-1)}$ for $y$ we have that also the following integral equation holds

$$
\left[V_{\phi_{1}^{(1)}} y^{(n-1)}\right](t)=y^{(n-1)}(t) \phi_{1}(t)-\left[V_{\phi_{1}} y^{(n)}\right](t) .
$$

By introducing the differential constraint (4) in (12) and by exploiting the linearity of modulation integrals, we obtain the intermediate expression

$$
\begin{aligned}
& {\left[V_{\phi_{1}^{(1)}} y^{(n-1)}\right](t)=y^{(n-1)}(t) \phi_{1}(t)} \\
& \quad-\sum_{i=0}^{n-1} a_{i}\left[V_{\phi_{1}} y^{(i)}\right](t)-\sum_{k=0}^{m-1} b_{k}\left[V_{\phi_{1}} u^{(k)}\right](t) .
\end{aligned}
$$

Substituting (10) and (11) in (13), after some cumbersome algebra the expression (13) can be rearranged as 
follows:

$$
\begin{aligned}
& (-1)^{n-1}\left[V_{\phi_{1}^{(n)}} y\right](t)+\sum_{i=0}^{n-1} a_{i}(-1)^{i}\left[V_{\phi_{1}^{(i)}} y\right](t) \\
& +\sum_{k=0}^{m-1} b_{k}(-1)^{k}\left[V_{\phi_{1}^{(k)}} u\right](t)=\sum_{r=0}^{n-1} \gamma_{\phi_{1}, r}(t) z_{r}(t)
\end{aligned}
$$

where $\gamma_{\phi_{1}, 0}(t), \ldots, \gamma_{\phi_{1}, r}(t), \ldots, \gamma_{\phi_{1}, n-1}(t)$ are known functions of time depending on the $\operatorname{MF} \phi_{1}(\cdot)$ and its derivatives:

$$
\gamma_{\phi_{1}, r}(t)=\phi_{1}^{(n-r-1)}(t)(-1)^{n-r-1}, r \in\{0, \ldots, n-1\} .
$$

Observing that all the terms on the left-hand side of (14) can be obtained by processing the signals $y$ and $u$ by modulation integrals, it is convenient to define the signal

$$
\begin{aligned}
v_{\phi_{1}}(t) \triangleq(-1)^{n-1}\left[V_{\phi_{1}^{(n)}} y\right](t)+\sum_{i=0}^{n-1} a_{i}(-1)^{i}\left[V_{\phi_{1}^{(i)}} y\right](t) \\
\quad+\sum_{k=0}^{m-1} b_{k}(-1)^{k}\left[V_{\phi_{1}^{(k)}} u\right](t) \\
=\int_{0}^{t} \sum_{i=0}^{n} \phi_{1}^{(i)}(\tau)\left(\check{a}_{i} y(\tau)+\check{b}_{i} u(\tau)\right) d \tau
\end{aligned}
$$

where $\check{a}_{i}=(-1)^{i} a_{i}, \forall i \in\{0, \ldots, n-1\}, \check{a}_{i}=(-1)^{n-1}, i=$ $n$, and $\breve{b}_{i}=(-1)^{i} b_{i}, \forall i \in\{0, \ldots, m-1\}, \breve{b}_{i}=0, \forall i \in$ $\{m, \ldots, n\}$. As such, (14) can be rewritten in a compact form, as follows

$$
v_{\phi_{1}}(t)=\sum_{r=0}^{n-1} \gamma_{\phi_{1}, r}(t) z_{r}(t)
$$

Note that the $n$ states $z_{0}(t), \ldots, z_{r}(t), \ldots, z_{n-1}(t)$ appear linearly in equation (17). On the other side, (17) cannot be directly inverted with respect to the unknown state variables, being rank-deficient. To solve this issue, it is possible to augment the number of constraints, forming a well posed system invertible with respect to the unknowns, by introducing further $(n-1)$ equations analogous to (17) constructed with different-unique MFs (see Definition 3.1), denoted by $\phi_{2}, \ldots, \phi_{n}$ in the sequel. Accordingly, we obtain the following time-varying algebraic linear system

$$
\boldsymbol{\Gamma}(t) \mathbf{z}(t)=\boldsymbol{v}(t)
$$

where $\boldsymbol{v}(t) \triangleq\left[v_{\phi_{1}}(t) \cdots v_{\phi_{h}}(t) \cdots v_{\phi_{n}}(t)\right]^{\top} \in \mathbb{R}^{n}$ is a vector of known signals (obtainable by processing the input-output signals through modulation integrals (16)) and $\boldsymbol{\Gamma}(t) \in \mathbb{R}^{n \times n}$ is a square time-varying matrix formed by MF dependent functions $\gamma_{\phi_{1}, r}(t)$ (see (15)):

$$
\boldsymbol{\Gamma}(t) \triangleq\left[\begin{array}{ccccc}
\gamma_{\phi_{1}, 0}(t) & \cdots & \gamma_{\phi_{1}, r}(t) & \cdots & \gamma_{\phi_{1}, n-1}(t) \\
\vdots & & \vdots & & \vdots \\
\gamma_{\phi_{h}, 0}(t) & \cdots & \gamma_{\phi_{h}, r}(t) & \cdots & \gamma_{\phi_{h}, n-1}(t) \\
\vdots & & \vdots & & \vdots \\
\gamma_{\phi_{n}, 0}(t) & \cdots & \gamma_{\phi_{n}, r}(t) & \cdots & \gamma_{\phi_{n}, n-1}(t)
\end{array}\right]
$$

In view of the linear relation (18) one can be tempted to obtain the full state vector by:

$$
\mathbf{z}(t)=(\boldsymbol{\Gamma}(t))^{-1} \boldsymbol{v}(t), \forall t>t_{\epsilon}
$$

where $t_{\epsilon}$ is a small time instant, provided that $\Gamma(t)$ is invertible for any $t>t_{\epsilon}$, so as to circumvent the singularity $\Gamma(0)=0$ due to $(9)$. Although $\boldsymbol{\Gamma}(t)$ can be made nonsingular by a proper choice of the MFs $\left\{\phi_{h}\right\}_{h \in\{1, \ldots, n\}}$, we will show in the following lines that the simple inversion (19) formula will not work without further provisions, being prone to diverge due to error accumulation, in particular when the estimator is run in a noisy environment. Indeed, recall (16) and assume that the output $y(t)$ is corrupted by a norm-bounded additive noise $d_{y}(t):\left|d_{y}(t)\right| \leq \bar{d}_{y}$. We denote by

$$
\hat{y}(t)=y(t)+d_{y}(t)
$$

the perturbed output signal and by $\hat{v}_{\phi_{h}}(t)$ the noisy counterpart of the signal $\breve{v}_{\phi_{h}}(t)$ defined in (16):

$$
\hat{v}_{\phi_{h}}(t)=\int_{0}^{t} \sum_{i=0}^{n} \phi_{h}^{(i)}(\tau)\left(\check{a}_{i}\left(y(\tau)+d_{y}(\tau)\right)+\check{b}_{i} u(\tau)\right) d \tau
$$

and the error signal $\tilde{v}_{\phi_{h}}(t)=\hat{v}_{\phi_{h}}(t)-v_{\phi_{h}}(t)$, which can be expressed by

$$
\tilde{v}_{\phi_{h}}(t)=\int_{0}^{t} d_{y}(\tau) \sum_{i=0}^{n} \check{a}_{i} \phi_{h}^{(i)}(\tau) d \tau \text {. }
$$

Unless the disturbance $d_{y}(\tau)$ is zero mean on $[0, t]$, and $d_{y}(\tau)$ and $\sum_{i=0}^{n} \check{a}_{i} \phi_{h}^{(i)}(\tau)$ are uncorrelated, the integral tends to diverge (for example, when $d_{y}$ is a deterministic perturbation like an offset in the measurement). This issue can be circumvented by using a strategy that we have called Periodic Rescaling, which is discussed the next Section 4 and analyzed in 5.

\section{Periodic Rescaling}

Let us now extend the notation in order to address the more generic case in which the modulation operators are 
allowed to begin integrating at a time instant $t_{0}$ different from 0 . Denoting by

$$
\boldsymbol{v}\left(t \mid t_{0}\right) \triangleq\left[v_{\phi_{1}}\left(t \mid t_{0}\right) \cdots v_{\phi_{h}}\left(t \mid t_{0}\right) \cdots v_{\phi_{n}}\left(t \mid t_{0}\right)\right]^{\top} \in \mathbb{R}^{n},
$$

the vector of time-shifted modulation integrals with:

$$
v_{\phi_{h}}\left(t \mid t_{0}\right) \triangleq \int_{t_{0}}^{t} \sum_{i=0}^{n} \phi_{h}^{(i)}\left(\tau-t_{0}\right)\left(\check{a}_{i} y(\tau)+\check{b}_{i} u(\tau)\right) d \tau
$$

then a time-shifted version of (18) can be written as:

$$
\boldsymbol{\Gamma}\left(t-t_{0}\right) \mathbf{z}(t)=\boldsymbol{v}\left(t \mid t_{0}\right), \quad \forall t \geq t_{0} .
$$

In order to proceed with the further analysis, we let $T_{r}$ and $T_{\Delta}$ be two positive scalars and then define the discrete-time sequence $t_{i}=T_{\Delta}+i T_{r}, \forall i \in \mathbb{Z}_{>0}$. Noting that (24) holds for a generic $t_{0}$, then the following two equations hold together, for all $t>t_{k}-T_{\Delta}, k \in \mathbb{Z}_{>0}$ :

$$
\begin{aligned}
& \boldsymbol{\Gamma}\left(t-\left(t_{k}-T_{\Delta}\right)\right) \mathbf{z}(t)=\boldsymbol{v}\left(t \mid t_{k}-T_{\Delta}\right), \\
& \boldsymbol{\Gamma}\left(t-\left(t_{k-1}-T_{\Delta}\right)\right) \mathbf{z}(t)=\boldsymbol{v}\left(t \mid t_{k-1}-T_{\Delta}\right) .
\end{aligned}
$$

It is worth to point out that the vector $\boldsymbol{v}\left(t \mid t_{k}-T_{\Delta}\right)$ contains the information collected in the time-window $\left[t_{k}-T_{\Delta}, t\right]$ (stored by integration), while the vector $\boldsymbol{v}\left(t \mid t_{k-1}-T_{\Delta}\right)$ contains information collected in the longer time-window $\left[t_{k-1}-T_{\Delta}, t\right]$. By using the constraint (25), it is possible to establish an algebraic relation between $\boldsymbol{v}\left(t \mid t_{k}-T_{\Delta}\right)$ and $\boldsymbol{v}\left(t \mid t_{k-1}-T_{\Delta}\right)$ that permits to discard old information carried by the data processed in the interval $\left[t_{k-1}-T_{\Delta}, t_{k}-T_{\Delta}\right]$ (see the scheme in Fig. 1). For $t=t_{k}$, in view of (25), we can write:

$$
\begin{aligned}
& \boldsymbol{v}\left(t_{k} \mid t_{k}-T_{\Delta}\right) \\
& \quad=\boldsymbol{\Gamma}\left(T_{\Delta}\right)\left(\boldsymbol{\Gamma}\left(t_{k}-t_{k-1}+T_{\Delta}\right)\right)^{-1} \boldsymbol{v}\left(t_{k} \mid t_{k-1}-T_{\Delta}\right) \\
& \quad=\boldsymbol{\Gamma}\left(T_{\Delta}\right)\left(\boldsymbol{\Gamma}\left(T_{r}+T_{\Delta}\right)\right)^{-1} \boldsymbol{v}\left(t_{k} \mid t_{k-1}-T_{\Delta}\right)
\end{aligned}
$$

that is, we can compute the vector $\boldsymbol{v}\left(t_{k} \mid t_{k}-T_{\Delta}\right)$ from the vector $\boldsymbol{v}\left(t_{k} \mid t_{k-1}-T_{\Delta}\right)$ through a simple algebraic rescaling. According to the above discussion, then $T_{r}$ is the time between two rescaling events, while $T_{\Delta}$ represents the length of the equivalent integration window after a each operation. In order to avoid error accumulation and windup of integrators, a condition for the design of $T_{r}$ and $T_{\Delta}$ is determined in the next section (see Theorem 5.1 ) on the basis of an ISS analysis of the observer subjected to periodic rescaling. In order to carry out the ISS analysis, we need to express the rescaling action in a formal way, modeling the observer with periodic rescaling as a jump-linear system.

In the sequel, for the sake of brevity, we let

$$
\xi_{h}(t) \triangleq v_{\phi_{h}}\left(t \mid t_{k-1}-T_{\Delta}\right), h \in\{1, \ldots, n\},
$$

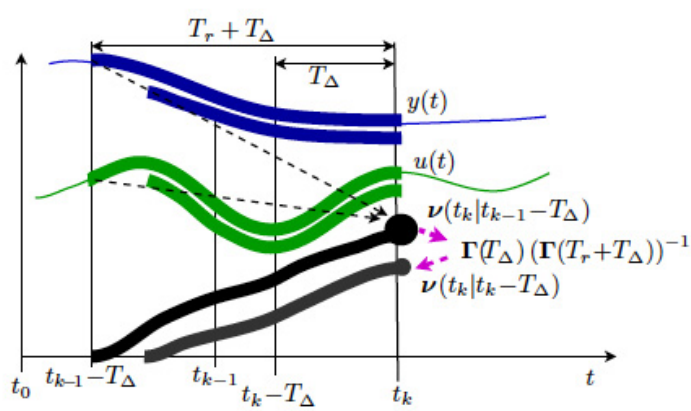

Fig. 1. The periodically rescaling scheme.

for all $t \in\left[t_{k-1}, t_{k}\right], k \in \mathbb{Z}_{>0}$, then it turns out that

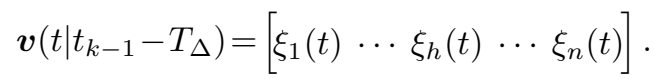

and

$$
\begin{aligned}
& \xi_{h}(t)= \\
& \int_{t_{k-1}-T_{\Delta}}^{t} \sum_{i=0}^{n} \phi_{h}^{(i)}\left(\tau-t_{k-1}+T_{\Delta}\right)\left(\check{a}_{i} y(\tau)+\check{b}_{i} u(\tau)\right) d \tau .
\end{aligned}
$$

In view of (26), the state undergoes a jump-transition at time $t_{k}$ satisfying the following constraint

$$
\xi_{h}\left(t_{k}^{+}\right)=\mathbf{e}_{h}^{\top} \boldsymbol{\Gamma}\left(T_{\Delta}\right)\left(\boldsymbol{\Gamma}\left(T_{r}+T_{\Delta}\right)\right)^{-1} \boldsymbol{v}\left(t_{k} \mid t_{k-1}-T_{\Delta}\right)
$$

where we denote by $\mathbf{e}_{h} \in \mathbb{R}^{n}$ the $h$-th canonical basis vector. In the subsequent time-window $t \in\left(t_{k}, t_{k+1}\right]$ the state variables will also verify the following equation:

$$
\xi_{h}(t)=\int_{t_{k}-T_{\Delta}}^{t} \sum_{i=0}^{n} \phi_{h}^{(i)}\left(\tau-t_{k}+T_{\Delta}\right)\left(\check{a}_{i} y(\tau)+\check{b}_{i} u(\tau)\right) d \tau .
$$

As such, the auxiliary variable $\xi_{h}$ evolves according to the following hybrid dynamics (jump-linear system):

$$
\left\{\begin{array}{l}
\dot{\xi}_{h}(t)=\sum_{i=0}^{n} \phi_{h}^{(i)}(t)\left(\check{a}_{i} y(t)+\check{b}_{i} u(t)\right), \quad 0 \leq t \leq t_{1} ; \\
\dot{\xi}_{h}(t)=\sum_{i=0}^{n} \phi_{h}^{(i)}\left(t-t_{k}+T_{\Delta}\right)\left(\check{a}_{i} y(t)+\check{b}_{i} u(t)\right), t_{k}<t \leq t_{k+1} ; \\
\xi_{h}\left(t_{k}^{+}\right)=\mathbf{e}_{h}^{\top} \boldsymbol{\Gamma}\left(T_{\Delta}\right) \boldsymbol{\Gamma}\left(T_{r}+T_{\Delta}\right)^{-1} \boldsymbol{v}\left(t_{k} \mid t_{k-1}-T_{\Delta}\right), t=t_{k} .
\end{array}\right.
$$

with $\xi_{h}(0)=0$. Finally, the state estimate of the canonical system (2) is given by (19), $\forall t \in\left(0, t_{1}\right]$, and for all $t: t_{k}<t \leq t_{k+1}, k \in \mathbb{Z}_{>0}$, it follows

$$
\mathbf{z}(t)=\left(\boldsymbol{\Gamma}\left(t-t_{k}+T_{\Delta}\right)\right)^{-1} \boldsymbol{v}\left(t \mid t_{k}-T_{\Delta}\right)
$$




\section{Robustness Analysis}

In this section, we address the stability properties of the rescaling scheme in the presence of both input and output disturbances. Let us denote by $\hat{u}(t)=u(t)+d_{u}(t)$ the available input with bounded noise $d_{u}(t):\left|d_{u}(t)\right| \leq$ $\bar{d}_{u}$, while the perturbed output $\hat{y}(t)$ is defined $(20)$. By introducing the state estimation error: $\tilde{\mathbf{z}}(t) \triangleq \hat{\mathbf{z}}(t)-$ $\mathbf{z}(t)$ and the so-called inner state error: $\tilde{\xi}_{h}(t) \triangleq \hat{\xi}_{h}(t)-$ $\xi_{h}(t), h=1, \cdots, n$, we derive from (29) the inner error dynamics with rescaling

$$
\left\{\begin{array}{c}
\dot{\tilde{\xi}}_{h}(t)=\sum_{i=0}^{n} \phi_{h}^{(i)}(t)\left(\check{a}_{i} d_{y}(t)+\check{b}_{i} d_{u}(t)\right), \quad 0 \leq t \leq t_{0} \\
\dot{\tilde{\xi}}_{h}(t)=\sum_{i=0}^{n} \phi_{h}^{(i)}\left(t-t_{k}+T_{\Delta}\right)\left(\check{a}_{i} d_{y}(t)+\check{b}_{i} d_{u}(t)\right), \\
t_{k}<t \leq t_{k+1} \\
\dot{\tilde{\xi}}_{h}\left(t_{k}^{+}\right)=\mathbf{e}_{h}^{\top} \boldsymbol{\Gamma}\left(T_{\Delta}\right) \boldsymbol{\Gamma}\left(T_{r}+T_{\Delta}\right)^{-1} \tilde{\boldsymbol{v}}\left(t_{k} \mid t_{k-1}-T_{\Delta}\right), t=t_{k}
\end{array}\right.
$$

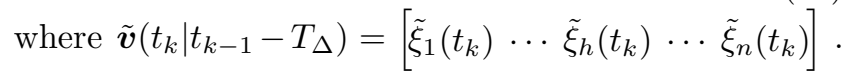
The state estimation error $\tilde{\mathbf{z}}(t)$ can be obtained from the inner state error $\tilde{\xi}(t)$ by projecting $\tilde{\boldsymbol{v}}$ through the linear map $\boldsymbol{\Gamma}^{-1}$ as done in (30). The following result enables us to guarantee the stability of the state observer in the presence of the disturbances $d_{u}(t)$ and $d_{y}(t)$.

Theorem 5.1 Given the SISO system (1), assume the positive constants $T_{r}$ and $T_{\Delta}$ are designed, such that the following inequality is verified:

$$
\left|\left(\boldsymbol{\Gamma}\left(T_{\Delta}\right)\left(\boldsymbol{\Gamma}\left(T_{r}+T_{\Delta}\right)\right)^{-1}\right)\right|^{2} e^{2 T_{r}}<1
$$

Then, the state estimation error $\tilde{\mathbf{z}}(t)$ produced by the observer (29), (19) and (30) is ISS with respect to bounded perturbations $d_{u}(t), d_{y}(t):\left|d_{u}(t)\right| \leq \bar{d}_{u},\left|d_{y}(t)\right| \leq \bar{d}_{y}$, with arbitrary and finite $\bar{d}_{u} \in \mathbb{R}_{\geq 0}, \bar{d}_{y} \in \mathbb{R}_{\geq 0}$.

Proof. Let us introduce first the auxiliary functions: $V_{h}(t)=\tilde{\xi}_{h}(t)^{2}, \forall h=1, \cdots, n$, that will be used later to define a Lyapunov function for the error system. Then the derivative of $V_{h}$ along the inner state error trajectory in the interval $t_{k}<t \leq t_{k+1}$ satisfies,

$$
\begin{aligned}
\dot{V}_{h}(t) \leq & 2 \sum_{i=0}^{n}\left|\phi_{h}^{(i)}\left(t-t_{k}+T_{\Delta}\right)\right|\left|\tilde{\xi}_{h}(t)\right|\left(\left|\check{a}_{i}\right| \bar{d}_{y}+\left|\check{b}_{i}\right| \bar{d}_{u}\right) \\
\leq & 2\left|\tilde{\xi}_{h}(t)\right|^{2}+\left(\sum_{i=0}^{n}\left|\phi_{h}^{(i)}\left(t-t_{k}+T_{\Delta}\right)\right|\left|\check{a}_{i}\right|\right)^{2} \bar{d}_{y}^{2} \\
& +\left(\sum_{i=0}^{n}\left|\phi_{h}^{(i)}\left(t-t_{k}+T_{\Delta}\right)\right|\left|\check{b}_{i}\right|\right)^{2} \bar{d}_{u}^{2} \\
= & 2 V_{h}(t)+\sigma_{h}\left(\bar{d}_{u}, \bar{d}_{y}\right), \quad t_{k}<t \leq t_{k}+1
\end{aligned}
$$

where

$$
\begin{aligned}
\sigma_{h}\left(\bar{d}_{u}, \bar{d}_{y}\right) \triangleq & \left(\sum_{i=0}^{n} \sup _{0<\tau \leq T_{r}+T_{\Delta}}\left|\phi_{h}^{(i)}(\tau)\right|\left|\check{a}_{i}\right|\right)^{2} \bar{d}_{y}^{2} \\
& +\left(\sum_{i=0}^{n} \sup _{0<\tau \leq T_{r}+T_{\Delta}}\left|\phi_{h}^{(i)}(\tau)\right|\left|\check{b}_{i}\right|\right)^{2} \bar{d}_{u}^{2}
\end{aligned}
$$

By the Gronwall-Bellman Lemma, each function $V_{h}(t)$ can be bounded as follows:

$$
\begin{array}{r}
V_{h}(t) \leq V_{h}\left(t_{k}^{+}\right) e^{2\left(t-t_{k}\right)}+\frac{1}{2}\left(e^{2\left(t-t_{k}\right)}-1\right) \sigma_{h}\left(\bar{d}_{u}, \bar{d}_{y}\right) \\
\forall t \in\left(t_{k}, t_{k+1}\right], \forall k \in \mathbb{Z}_{>0}
\end{array}
$$

Substituting $t_{k+1}$ for $t$ in (33), we can establish the bound at $t_{k+1}$

$$
V_{h}\left(t_{k+1}\right) \leq V_{h}\left(t_{k}^{+}\right) e^{2 T_{r}}+e^{2 T_{r}} \sigma_{h}\left(\bar{d}_{u}, \bar{d}_{y}\right)
$$

Due to the hybrid dynamics of the inner state error (see (31)), then also the function $V_{h}$ undergoes a jump transition at time $t_{k+1}: V_{h}\left(t_{k+1}^{+}\right)=\tilde{\xi}_{h}\left(t_{k+1}^{+}\right)^{2}$. In order to determine the value of $V_{h}\left(t_{k+1}^{+}\right)$, let us first study the bound of $\tilde{\boldsymbol{v}}\left(t_{k+1}^{+} \mid t_{k-1}-T_{\Delta}\right)$, which is the vector collecting all $\tilde{\xi}_{h}\left(t_{k+1}^{+}\right), \forall h=1,2, \cdots, n$ :

$$
\begin{aligned}
\left|\tilde{\boldsymbol{v}}\left(t_{k+1}^{+} \mid t_{k-1}-T_{\Delta}\right)\right| & \leq\left|\boldsymbol{\Gamma}\left(T_{\Delta}\right) \boldsymbol{\Gamma}\left(T_{r}+T_{\Delta}\right)^{-1}\right| \\
& \times\left|\tilde{\boldsymbol{v}}\left(t_{k+1} \mid t_{k}-T_{\Delta}\right)\right|, k \in \mathbb{Z}_{>0}
\end{aligned}
$$

where

$$
\left|\tilde{\boldsymbol{v}}\left(t_{k+1} \mid t_{k}-T_{\Delta}\right)\right|=\sqrt{\sum_{h=1}^{n} \tilde{\xi}_{h}\left(t_{k+1}\right)^{2}}=\sqrt{\sum_{h=1}^{n} V_{h}\left(t_{k+1}\right)} .
$$

In view of $(34)$, we have that

$$
\left|\tilde{\boldsymbol{v}}\left(t_{k+1} \mid t_{k}-T_{\Delta}\right)\right| \leq \sqrt{e^{2 T_{r}} \sum_{h=1}^{n}\left(V_{h}\left(t_{k}^{+}\right)+\sigma_{h}\left(\bar{d}_{u}, \bar{d}_{y}\right)\right)},
$$

thus resulting in a further bound on $\left|\tilde{\boldsymbol{v}}\left(t_{k+1}^{+} \mid t_{k}-T_{\Delta}\right)\right|$ :

$$
\begin{gathered}
\left|\tilde{\boldsymbol{v}}\left(t_{k+1}^{+} \mid t_{k-1}-T_{\Delta}\right)\right| \leq \kappa \sqrt{e^{2 T_{r} \sum_{h=1}^{n}\left(V_{h}\left(t_{k}^{+}\right)+\sigma_{h}\left(\bar{d}_{u}, \bar{d}_{y}\right)\right)}} \\
\Longrightarrow \sqrt{\sum_{h=1}^{n} V_{h}\left(t_{k+1}^{+}\right)} \leq \kappa \sqrt{e^{2 T_{r} \sum_{h=1}^{n}\left(V_{h}\left(t_{k}^{+}\right)+\sigma_{h}\left(\bar{d}_{u}, \bar{d}_{y}\right)\right)}}
\end{gathered}
$$

where, for the sake of brevity, we have posed $\kappa \triangleq\left|\boldsymbol{\Gamma}\left(T_{\Delta}\right) \boldsymbol{\Gamma}\left(T_{r}+T_{\Delta}\right)^{-1}\right|$. 
Finally, let $V(t)=\sum_{h=1}^{n} V_{h}(t)$ be the Lyapunov function candidate for the inner state error dynamics. Then we can guarantee the following difference bound on the discrete Lyapunov function sequence, sampled just after each state jump:

$$
V\left(t_{k+1}^{+}\right) \leq \kappa^{2} e^{2 T_{r}} V\left(t_{k}^{+}\right)+\kappa^{2} e^{2 T_{r}} \sum_{h=1}^{n} \sigma_{h}\left(\bar{d}_{u}, \bar{d}_{y}\right)
$$

which can be rearranged in the following compact form

$$
V\left(t_{k+1}^{+}\right)-V\left(t_{k}^{+}\right) \leq-\left(1-\kappa^{2} e^{2 T_{r}}\right) V\left(t_{k}^{+}\right)+\kappa^{2} e^{2 T_{r}} \sigma\left(\bar{d}_{u}, \bar{d}_{y}\right)
$$

with $\sigma\left(\bar{d}_{u}, \bar{d}_{y}\right) \triangleq n \max _{h=1, \cdots, n}\left\{\sigma_{h}\left(\bar{d}_{u}, \bar{d}_{y}\right)\right\}$

Thanks to (32), the designed $T_{r}$ and $T_{\Delta}$ enforce $\kappa^{2} e^{2 T_{r}}<$ 1 , which implies that $V$ is a discrete ISS Lyapunov function for the sampled inner state error system. Therefore, $\tilde{\xi}_{h}(t)$ is ISS with respect to $\bar{d}_{u}$ and $\bar{d}_{y}$. Finally, it is straightforward to show that the state estimation error subsumes the bound

$$
|\tilde{\mathbf{x}}(t)| \leq\left|\mathbf{T}^{-1}\right| \sup _{0<\tau \leq T_{r}+T_{\Delta}}\left|\boldsymbol{\Gamma}(\tau)^{-1}\right| \sum_{h=1}^{n}\left|\tilde{\xi}_{h}(t)\right|,
$$

\section{Instances of Admissible Modulating Func- tions}

In this section, we propose two instances of MFs (that fulfill the requirements (9)), which are employed later on in the simulations. Moreover, the influence of the MFs' coefficients on the invertibility of $\boldsymbol{\Gamma}(t)$ is investigated to get more insight into the proper setting of design parameters. To this end, we will analyse the condition number $^{1}$ of the $\Gamma(t)$ matrix for different types of MFs.

\section{1) Exponential modulating function:}

$$
\phi_{h}(t)=\left(1-e^{-\rho_{h} t}\right)^{n}, h \in\{1, \ldots, n\}
$$

which is parametrized by a set of constants $\rho_{h} \in \mathbb{R}_{>0}$ with a constraint $\rho_{i} \neq \rho_{j}$, for $i \neq j$.

Now, let us focus on the case $n=2$ and consider two exponential MFs $\phi_{h}(t), h=1,2$, which gives rise to

$$
\boldsymbol{\Gamma}(t)=\left[\begin{array}{cc}
-2 \rho_{1} e^{-\rho_{1} t}\left(1-e^{-\rho_{1} t}\right) & \left(1-e^{-\rho_{1} t}\right)^{2} \\
-2 \rho_{2} e^{-\rho_{2} t}\left(1-e^{-\rho_{2} t}\right) & \left(1-e^{-\rho_{2} t}\right)^{2}
\end{array}\right]
$$

1 Let $\left|\frac{\lambda_{\max }(\Gamma(t))}{\lambda_{\min }(\Gamma(t))}\right|$ denote the instantaneous condition number of $\boldsymbol{\Gamma}(t)$ with $\lambda_{\min }(\boldsymbol{\Gamma}(t)), \lambda_{\max }(\boldsymbol{\Gamma}(t))$ the minimum and maximum eigenvalue of matrix $\boldsymbol{\Gamma}(t)$, respectively.
The invertibility of $\boldsymbol{\Gamma}(t)$ is implied by

$$
\begin{array}{r}
\operatorname{det}(\boldsymbol{\Gamma}(t))=2\left(1-e^{-\rho_{1} t}\right)\left(1-e^{-\rho_{2} t}\right)\left[\left(1-e^{-\rho_{1} t}\right) \rho_{2} e^{-\rho_{2} t}\right. \\
\left.-\left(1-e^{-\rho_{2} t}\right) \rho_{1} e^{-\rho_{1} t}\right] \neq 0
\end{array}
$$

which holds if the following condition is verified

$$
\left(1-e^{-\rho_{1} t}\right) \rho_{2} e^{-\rho_{2} t} \neq\left(1-e^{-\rho_{2} t}\right) \rho_{1} e^{-\rho_{1} t} .
$$

Indeed, by applying the $\ln (\cdot)$ operator on both sides of $(39)$, we obtain $\rho_{2} t \neq \rho_{1} t \rightarrow\left(\rho_{1}-\rho_{2}\right) t \neq 0$. For any designed $T_{r}, T_{\Delta}, \rho_{1}, \rho_{2}$, this condition is verified as $\rho_{i} \neq \rho_{j}$, for $i \neq j$, and therefore it implies $\operatorname{det}(\boldsymbol{\Gamma}(t)) \neq$ $0, \forall t \in\left(0, T_{r}+T_{\Delta}\right]$. In Fig. 2 , the condition numbers of $\boldsymbol{\Gamma}(t), \forall t \geq 0.3$ are shown for three pairs of $\rho_{1}, \rho_{2}$.
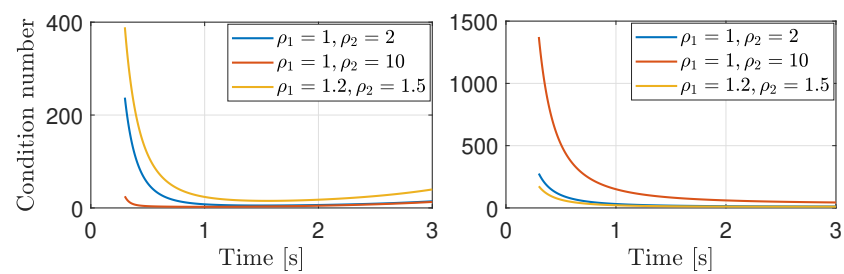

Fig. 2. Time behavior of the condition numbers of $\boldsymbol{\Gamma}(t)$ formed by exponential (left) and time-monomial (right) MFs.

2) Time-monomial modulating function:

$$
\phi_{h}(t)=\frac{\rho_{h} t^{2 n-h}}{(2 n-h) !}, \quad h \in\{1, \ldots, n\}
$$

with $\rho_{h} \in \mathbb{R}_{>0}$ arbitrary (uniqueness does not apply here). Although $\phi_{h}(t)$ and its derivatives are monotonically increasing functions with respect to $t$, they are only computed over the time interval $\left[0, T_{r}+T_{\Delta}\right]$ due to the periodic rescaling, thus circumventing the issue of numerical overflow. Following the same steps taken above, we obtain

$$
\operatorname{det}(\boldsymbol{\Gamma}(t))=-\frac{\rho_{1} \rho_{2} t^{4}}{12} \neq 0
$$

under the scenario that $n=2$. Thus verifying the invertibility of $\boldsymbol{\Gamma}(t)$ over a time interval $\left(0, T_{r}+T_{\Delta}\right]$ for any positive parameters $T_{r}, T_{\Delta}, \rho_{1}, \rho_{2}$.

As can be noticed from Fig. 2, the matrix $\boldsymbol{\Gamma}(t)$ has a large condition number at the very beginning. In this respect, the parameters $\rho_{h}, h=1,2, \cdots, n$ must be chosen to minimize the condition number, in order to render feasible the practical inversion of $\boldsymbol{\Gamma}(t)$ in the interval $\left[t_{\epsilon}, T_{\Delta}+T_{r}\right]$. Note that the inverse matrix $\boldsymbol{\Gamma}(t)^{-1}$ can be computed analytically once the MFs have been chosen and the inverse can be implemented directly, to avoid online matrix inversion.

\section{Simulation Results}

Example 1 In this example, we compare the proposed method with the impulsive observer reported in [13]. 
Consider a spring mass system used in [13], which is described by the state-space realization (1) with

$$
\mathbf{A}=\left[\begin{array}{cccc}
0 & 0 & 1 & 0 \\
0 & 0 & 0 & 1 \\
-2 & 1 & -1 & 0 \\
2 & -2 & 0 & -2
\end{array}\right], \mathbf{b}=\left[\begin{array}{l}
0 \\
0 \\
1 \\
0
\end{array}\right], \mathbf{c}=\left[\begin{array}{l}
1 \\
0 \\
0 \\
0
\end{array}\right]
$$

$u(t)=\sin (t)$ and $x_{0}=\left[\begin{array}{llll}5 & -2 & 3 & 4\end{array}\right]^{\top}$. The method [13] is tuned by the same parameters shown in [13], while we set: $\rho_{1}=1, \rho_{2}=2, \rho_{3}=3, T_{r}=T_{\Delta}=1$ s for the proposed observer with respect to time-monomial MFs. The convergence time is chosen as 1 s (i.e., $t_{\epsilon}=1 \mathrm{~s}$ ), identical to the value given in [13]. The simulation results are shown in Fig. 3, where both observers succeed in estimating the three inaccessible states $\left(x_{0}(t)\right.$ is available from the measurable output $y(t))$ within a predetermined finite time.
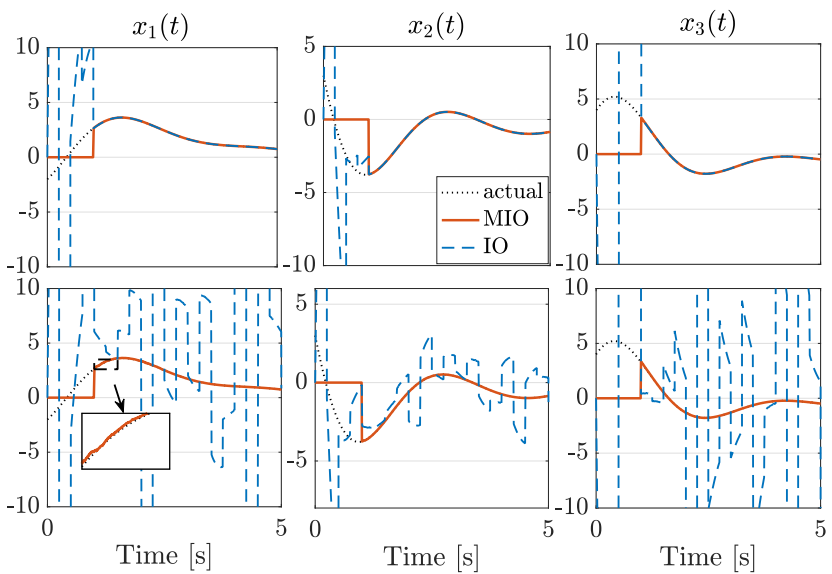

Fig. 3. Comparison between the proposed modulation integral observer (MIO) and the impulsive observer (IO) [13] in the noise-free (first row) and noisy (second row) conditions.

Next, the behavior of both observers in the presence output disturbance is examined by assuming an output measurement disturbance $d(t)$ that is formulated as a uniformly distributed random signal in the interval $[-0.1,0.1]$. It can be observed from Fig. 3 that the impulsive observer [13] is susceptible to the measured noise, while the proposed method offers strong attenuation of high-frequency noise, and yields accurate estimates in the noisy environment.

Example 2 The present example is aimed at comparing the proposed modulation integral observer with its reduced order counterpart [19]. Consider the canonical third-order system taken from [19]:

$$
\left\{\begin{array}{l}
\mathbf{z}^{(1)}(t)=\mathbf{A}_{z} \mathbf{z}(t)+\mathbf{b}_{z}(u(t)+d(t)), \\
y(t)=\mathbf{c}_{z}^{\top} \mathbf{z}(t)+d(t), \quad t \in \mathbb{R}_{\geq 0}
\end{array}\right.
$$

with

$$
\mathbf{A}_{z}=\left[\begin{array}{ccc}
-0.21 & 1 & 0 \\
-9.012 & 0 & 1 \\
-0.0901 & 0 & 0
\end{array}\right], \mathbf{b}_{z}=\left[\begin{array}{l}
0 \\
1 \\
1
\end{array}\right], \mathbf{c}_{z}=\left[\begin{array}{l}
1 \\
0 \\
0
\end{array}\right]
$$

where $z(0)=[1-101]$. The nominal input is $u(t)=$ $10 \sin 10 t+\sin 2 t$, however, $y(t)$ and $u(t)$ are both corrupted by a disturbance $d(t)$ that is subject to uniformly distribution within the interval $[-0.2,0.2]$. The proposed observer is designed by using exponential MFs with $\rho_{1}=1, \rho_{2}=2, \rho_{3}=3$. Both methods are activated at $0.5 \mathrm{~s}$ with $T_{r}=T_{\Delta}=1 \mathrm{~s}$.

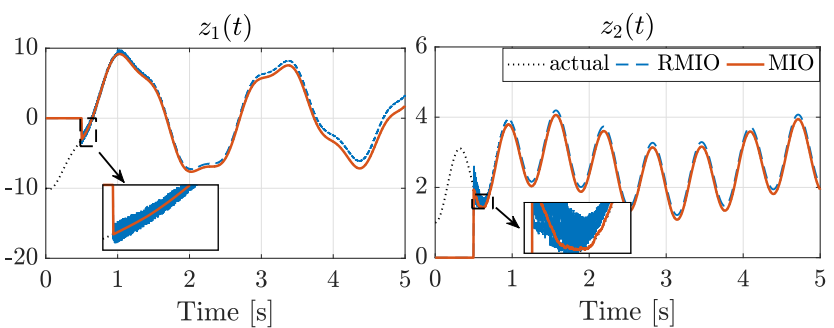

Fig. 4. Comparison between the proposed modulation integral observer (MIO) and the reduced order modulation integral observer (RMIO) [19] in the noisy condition.

It follows from Fig. 4 that in the presence of disturbances the transient and steady state accuracy are significantly improved by the proposed algorithm at the cost of one more dynamic order.

Example 3 This example aims at comparing the proposed method with the recent finite-time observer presented in [16] by using the system (41). The observer [16] is tuned to converge at $t=1 \mathrm{~s}$. From the results reported in Fig. 5, the convergence of the observed state to the real state of the linear system is guaranteed in a finite and fixed time. Compared to the behavior of the proposed method shown in Fig. 4, the approach designed in [16] is slightly more sensitive to the additive noise. Next, we further reduce the pre-determined converge time of [16] to $0.5 s$, identical to the value set for the proposed method in the previous example (see Fig. 4). As it can be seen, the converge speed is increased at the price of higher noise-sensitivity and larger overshoots. Such a trade-off does not arise in the methodology presented in the paper.

\section{Concluding Remarks}

The present paper is concerned with the design of a deadbeat state observer for SISO linear systems without high gain injection and with lower complexity compared to the kernel-based observer proposed by the authors in [18]. The method consists in processing the input and output measurements by modulation integrals with periodic rescaling, which aims to discard old information 


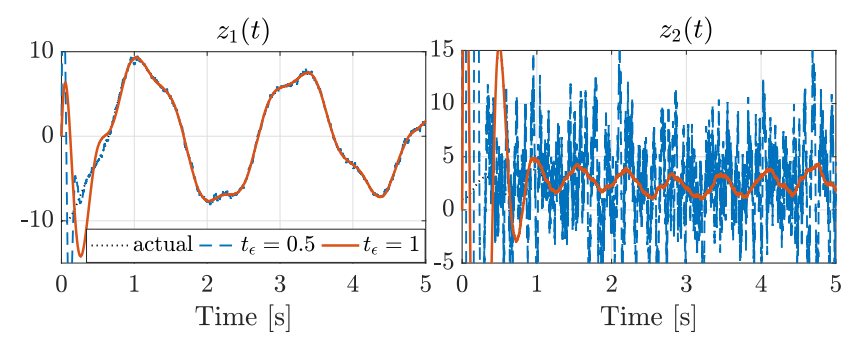

Fig. 5. State estimation of [16] in the noisy condition with different convergence time: $t_{\epsilon}=0.5 \mathrm{~s}$ and $t_{\epsilon}=1 \mathrm{~s}$.

at pre-determined discrete-time instants. The resulting observer is ISS with respect to bounded additive measurement perturbations.

\section{References}

[1] D. Luenberger, "Observers for multivariable systems," IEEE Trans. on Automatic Control, vol. 11, pp. 190-196, 1966.

[2] R. Kalman and R. Bucy, "New results inlinear filtering and prediction theory," J. of Basic Engrg., Trans. ASME, Series $D$, vol. 83, pp. 95-108, 1961.

[3] F. J. Bejarano, L. Fridman, and A. Pisano, "Global hierarchical observer for linear systems with unknown inputs," in Proc. of IEEE Conference on Decision and Control, Cancun, 2008, pp. 1883-1888.

[4] J. Davila, L. Fridman, and A. Levant, "Second-order slidingmode observer for mechanical systems," IEEE Trans. on Automatic Control, vol. 50, no. 11, pp. 1785-1789, 2005.

[5] D. Efimov and L. Fridman, "Global sliding-mode observer with adjusted gains for locally lipschitz systems," Automatica, vol. 47, no. 3, pp. 565-570, 2011.

[6] X. Yu, P. Li and Y. Zhang, "The Design of FixedTime Observer and Finite-Time Fault-Tolerant Control for Hypersonic Gliding Vehicles," IEEE Transactions on Industrial Electronics, vol. 65, no. 5, pp. 4135-4144, 2018.

[7] T. Menard, E. Moulay and W. Perruquetti, "Fixedtime observer with simple gains for uncertain systems," Automatica, vol. 81, pp. 438-446, 2017.

[8] Haik Silm and Rosane Ushirobira and Denis Efimov and JeanPierre Richard and Wim Michiels, "A note on distributed finite-time observers," IEEE Transactions on Automatic Control, 2018.

[9] F. L.-Ramirez, A. Polyakov, D. Efimov and W. Perruquetti, "Finite-time and fixed-time observer design: Implicit Lyapunov function approach," Automatica, vol. 87, pp. 5260, 2018.

[10] A. Medvedev and T. Toivonen, "Feedforward time-delay structures in state estimation: finite memory smoothing and continuous deadbeat observers," IEE Proceedings of Control Theory and Applications, vol. 141, pp. 121-129, 1994.

[11] R. Engel and G. Kresselmeier, "A continuous-time observer which converges in finite-time," IEEE Trans. Automatic Control, vol. 47, pp. 1202-1204, 2002.

[12] T. Raff and F. Allgöver, "Observers with impulsive dynamical behavior for linear and nonlinear continuous-time systems," in Proc. IEEE Conf. Decision and Control, New Orleans, 2007, pp. 4287-4292.

[13] T. Raff and F. Allgöver, "An observer that converges in finite time due to measurement-based state updates," in Proc. of the 17th IFAC World Congress, 2008, pp. 2693-2695.
[14] S. Han, W. Kwon, and P. Kim, "Receding-horizon unbiased FIR filters for continuous-time state-space models wihout a priori state inforations," IEEE Transactions on Automatic Control, vol. 46, pp. 766-770, 2001.

[15] W. Byrski, "The Survey of exact and optimal state observers in Hilbert spaces," in Proc. of the 2003 European Control Conference, Cambridge, UK, 2003.

[16] F. Lopez-Ramirez, A. Polyakov, D. Efimov, and W. Perruquetti, "Finite-time and fixed-time observers design via implicit Lyapunov function," in Proc. of the IEEE European Control Conference, Linz, 2015.

[17] A. Polyakov, D. Efimov, and W. Perruquetti, "Finite-time and fixed-time stabilization: Implicit Lyapunov function approach," Automatica, vol. 51, pp. 332-340, 2015.

[18] G. Pin, M. Lovera, A. Assalone, and T. Parisini, "Kernel-Based Non-Asymptotic State Estimation for Linear Continuous-Time Systems," in Proc. of the 2013 American Control Conference, Washington, DC, 2013, pp. 3129-3134.

[19] G. Pin, B. Chen, and T. Parisini, "The Modulation Integral Observer for Linear Continuous-Time Systems," in Proc. of the 2015 European Control Conference, Linz, Austria, pp. 2937-2944, 2015.

[20] J. Jouffroy and J. Reger, "Finite-time simultaneous parameter and state estimation using modulating functions," in Proc. of the IEEE Conference on Control Applications (CCA), pp. 394-399, 2015.

[21] Q. Guo, W. Perruquetti, and D. Efimov, "Universal Robust Adaptive Control of Robot Manipulators Using Real Time Estimation," IFAC-PapersOnLine, vol. 51, pp. 499-504, 2015. 